\title{
CORRECTION
}

\section{Correction: Genome sequence of an Australian kangaroo, Macropus eugenii, provides insight into the evolution of mammalian reproduction and development}

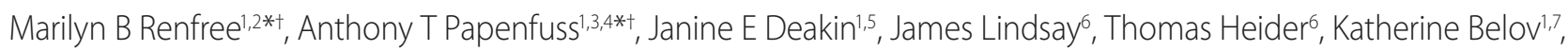
Willem Rens ${ }^{8}$, Paul D Waters, ${ }^{1,5}$, Elizabeth A Pharo², Geoff Shaw'1,2, Emily SW Wong ${ }^{1,7}$, Christophe M Lefèvre9 Kevin R Nicholas ${ }^{9}$, Yoko Kuroki10, Matthew J Wakefield ${ }^{1,3}$, Kyall R Zenger ${ }^{1,11,7}$, Chenwei Wang ${ }^{1,7}$, Malcolm Ferguson-Smith ${ }^{8}$,

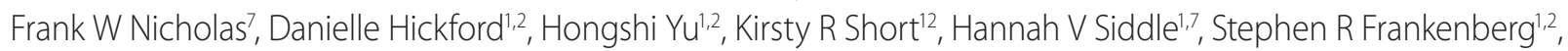
Keng Y Chew ${ }^{1,2}$, Brandon R Menzies ${ }^{1,13,2}$, Jessica M Stringer, ${ }^{1,2}$, Shunsuke Suzuki ${ }^{1,2}$, Timothy A Hore ${ }^{1,14}$, Margaret L Delbridge 1,5, Hardip R Patel ${ }^{1,5}$, Amir Mohammadi 1,5, Nanette Y Schneider ${ }^{1,2,15}$, Yanqiu Hu',2, William O'Hara ${ }^{6}$, Shafagh Al Nadaf1,5, Chen Wu', Zhi-Ping Feng ${ }^{16,3}$, Benjamin G Cocks ${ }^{17}$, Jianghui Wang ${ }^{17}$, Paul Flicek ${ }^{18}$, Stephen MJ Searle ${ }^{19}$, Susan Fairley ${ }^{19}$, Kathryn Beal18, Javier Herrero ${ }^{18}$, Dawn M Carone ${ }^{6,20}$, Yutaka Suzuki ${ }^{21}$, Sumio Sugano ${ }^{21}$, Atsushi Toyoda ${ }^{22}$,

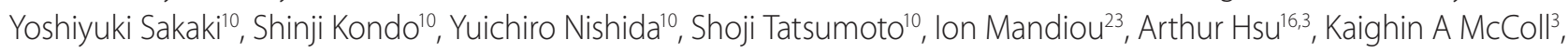
Benjamin Lansdell ${ }^{3}$, George Weinstock ${ }^{24}$, Elizabeth Kuczek',26,25, Annette McGrath25, Peter Wilson ${ }^{25}$, Artem Men²5, Mehlika Hazar-Rethinam²5, Allison Hall25, John Davis ${ }^{25}$, David Wood ${ }^{25}$, Sarah Williams ${ }^{25}$, Yogi Sundaravadanam², Donna M Muzny ${ }^{24}$, Shalini N Jhangiani24, Lora R Lewis ${ }^{24}$, Margaret B Morgan ${ }^{24}$, Geoffrey O Okwuonu ${ }^{24}$, San J Ruiz ${ }^{24}$, Jireh Santibanez ${ }^{24}$, Lynne Nazareth ${ }^{24}$, Andrew Cree ${ }^{24}$, Gerald Fowler ${ }^{24}$, Christie L Kovar24, Huyen H Dinh ${ }^{24}$, Vandita Joshi ${ }^{24}$, Chyn Jing ${ }^{24}$, Fremiet Lara ${ }^{24}$, Rebecca Thornton ${ }^{24}$, Lei Chen ${ }^{24}$, Jixin Deng ${ }^{24}$, Yue Liư ${ }^{24}$, Joshua Y Shen ${ }^{24}$, Xing-Zhi Song ${ }^{24}$, Janette Edson ${ }^{25}$, Carmen Troon ${ }^{25}$, Daniel Thomas ${ }^{25}$, Amber Stephens ${ }^{25}$, Lankesha Yapa ${ }^{25}$, Tanya Levchenko25, Richard A Gibbs ${ }^{24}$, Desmond W Cooper ${ }^{1,28}$, Terence P Speed ${ }^{1,3}$, Asao Fujiyama ${ }^{22,27}$, Jennifer A M Graves ${ }^{1,5}$, Rachel J O'Neill ${ }^{6}$, Andrew J Pask ${ }^{6,1,2}$, Susan M Forrest ${ }^{1,25}$ and Kim C Worley ${ }^{24}$

\section{Correction}

While compiling the article describing the genome sequence of the tammar wallaby [1] one of the authors was inadvertently omitted from the author list. This author, Hardip Patel, has been included in the corrected author list above.

\section{Author details}

'The Australian Research Council Centre of Excellence in Kangaroo Genomics, Australia. ${ }^{2}$ Department of Zoology, The University of Melbourne, Melbourne, Victoria 3010, Australia. ${ }^{3}$ Bioinformatics Division, The Walter and Eliza Hall Institute of Medical Research, Parkville, Victoria 3052, Australia. ${ }^{4}$ Department of Mathematics and Statistics, The University of Melbourne, Melbourne,

\section{tEqual contributors}

*Correspondence: m.renfree@unimelb.edu.au; papenfuss@wehi.edu.au

2Department of Zoology, The University of Melbourne, Melbourne, Victoria 3010, Australia

${ }^{3}$ Bioinformatics Division, The Walter and Eliza Hall Institute of Medical Research, Parkville, Victoria 3052, Australia

Full list of author information is available at the end of the article
Victoria 3010, Australia. ${ }^{5}$ Research School of Biology, The Australian National University, Canberra, ACT 0200, Australia. ${ }^{6}$ Department of Molecular and Cell Biology, Center for Applied Genetics and Technology, University of Connecticut, Storrs, CT 06269, USA. ${ }^{7}$ Faculty of Veterinary Science, University of Sydney, Sydney, NSW 2006, Australia. ${ }^{8}$ Department of Veterinary Medicine, University of Cambridge, Madingley Rd, Cambridge, CB3 OES, UK. Institute for Technology Research and Innovation, Deakin University, Geelong, Victoria, 3214, Australia. ${ }^{10}$ RIKEN Institute, 1-7-22 Suehiro-cho, Tsurumi-ku, Yokohama, Kanagawa 230-0045, Japan. "School of Marine and Tropical Biology, James Cook University, Townsville, Queensland 4811, Australia. ${ }^{12}$ Department of Microbiology and Immunology, The University of Melbourne, Melbourne, Victoria 3010, Australia. ${ }^{13}$ Leibniz Institute for Zoo and Wildlife Research, Alfred-Kowalke-Str. 17, Berlin 10315, Germany. ${ }^{14}$ Laboratory of Developmental Genetics and Imprinting, The Babraham Institute, Cambridge, CB22 3AT, UK. ${ }^{15}$ Department of Molecular Genetics, German Institute of Human Nutrition, Potsdam-Rehbruecke, Arthur-Scheunert-Allee 114-116, 14558 Nuthetal, Germany. ${ }^{16}$ Department of Medical Biology, The University of Melbourne, Melbourne, Victoria 3010, Australia. ${ }^{17}$ Biosciences Research Division, Department of Primary Industries, Victoria, 1 Park Drive, Bundoora 3083, Australia. ${ }^{18}$ European Bioinformatics Institute, Wellcome Trust Genome Campus, Hinxton, Cambridge, CB10 1SD, UK. ${ }^{19}$ Wellcome Trust Sanger Institute, Wellcome Trust Genome Campus, Hinxton, Cambridge, CB10 1SD, UK. ${ }^{20}$ Department of Cell Biology, University of Massachusetts Medical School, Worcester, MA 01655, USA. ${ }^{21}$ Graduate School of Frontier Sciences, The University of Tokyo, Chiba 277-8560, Japan. ${ }^{22}$ National Institute of Genetics, Mishima, Shizuoka 411-8540, Japan. ${ }^{23}$ Department of Computer Science 
and Engineering, University of Connecticut, Storrs, CT 06269, USA. ${ }^{24}$ Human Genome Sequencing Center, Department of Molecular and Human Genetics Baylor College of Medicine, Houston, TX 77030, USA. ${ }^{25}$ Australian Genome Research Facility, Melbourne, Victoria, 3052 and the University of Queensland St Lucia, Queensland 4072, Australia. ${ }^{26}$ Westmead Institute for Cancer

Research, University of Sydney, Westmead, New South Wales 2145, Australia.

${ }^{27}$ National Institute of Informatics, 2-1-2 Hitotsubashi, Chiyoda-ku, Tokyo 1018430, Japan. ${ }^{28}$ Department of Biological, Earth and Environmental Sciences, The University of New South Wales, Sydney, NSW 2052, Australia

Published: 30 December 2011

\section{Reference}

1. Renfree MB, Papenfuss AT, Deakin JE, Lindsay J, Heider T, Belov K, Rens W, Waters PD, Pharo EA, Shaw G, Wong ESW, Lefevre CM, Nicholas KR, Kuroki Y,
Wakefield MJ, Zenger KR, Wang C, Ferguson-Smith M, Nicholas FW, Hickford D, Yu H, Short KR, Siddle HV, Frankenberg SR, Chew KY, Menzies BR, Stringer JM, Suzuki S, Hore TA, Delbridge ML et al:: Genome sequence of an Australian kangaroo, Macropus eugenii, provides insight into the evolution of mammalian reproduction and development. Genome Biology 2011, 12:R81.

doi:10.1186/gb-2011-12-12-414

Cite this article as: Renfree MB, et al:: Correction: Genome sequence of an Australian kangaroo, Macropus eugenii, provides insight into the evolution of mammalian reproduction and development. Genome Biology 2011, 12:414. 